continuously emitted, annual health checkups focusing on the respiratory system were performed from February 2006. Since 2008 health checkups, a fractional exhaled nitric oxide concentration (FeNO) was measured. The present study aims to investigate the relationship between $\mathrm{SO}_{2}$ exposure and $\mathrm{FeNO}$ by comparing FeNO of the Miyakejima students with FeNO of reference students in another island in Japan, where $\mathrm{SO}_{2}$ concentration is far lower than the Japanese Environmental Standards.

Methods All subjects aged 13-14 years. Total number of Miyakejima and reference students were 83 and 31. From $\mathrm{SO}_{2}$ concentration measured at the fixed-point monitoring stations, we calculated the average $\mathrm{SO}_{2}$ concentration (ppb) during the 3 month periods prior to the health checkups. Based on the clinical practice guidelines of the Official American Thoracic Society, $35 \mathrm{ppb}$ was used as a cutoff point of FeNO. Results The average $\mathrm{SO}_{2}$ concentrations from 2008 to 2014 were $17.6,17.5,9.6,7.3,8.4,4.2$, and 5.0, respectively. The prevalence (\%) of Miyakejima students with abnormal FeNO at 2008 to 2014 health checkups 50.0, 27.3, 18.2, 57.1, 46.2, 32 38.5, and 50.0, respectively. These prevalence were not statistically higher than the prevalence of the reference students $(41.9 \%)$.

Discussion No clear dose-response relationship was observed in this study. In addition to this study, we also examined the medical records of patients at the Chuo-Clinic between 2005 and 2010. Totally 4586 medical records were examined. Of these, 2594 patients developed a disease potentially caused by $\mathrm{SO}_{2}$ concentration. Therefore, further studies with regards to the relationship between the incidence of disease and $\mathrm{SO}_{2}$ should be conducted.

\section{BERYLLIUM IN GOLF CLUBS: CASE REPORT}

${ }^{1,2}$ AA Khafagy ${ }^{*},{ }^{1} \mathrm{R}$ Harrison. 'University of California San Francisco, Division of Occupational and Environmental Medicine, San Francisco, USA; ${ }^{2}$ Umm Al-Qura University, College of Medicine, Department of Community Medicine and Pilgrims Healthcare, Makkah, Saudi Arabia

\subsection{6/oemed-2018-ICOHabstracts.1271}

Introduction Beryllium Sensitisation (BeS) is a delayed-type hypersensitivity to beryllium that can develop in susceptible workers exposed to beryllium. In some cases BeS progress to Chronic Beryllium Disease (CBD), which is marked by lymphocytic alveolitits and epitheloid granuloma in the lung parenchyma.

Case description A 53-year-old non-smoker male with medical history of diabetes mellitus type II and hyperlipidemia presented to his physician with progressive fatigue, weakness, and persistent cough. Three months later he developed dyspnea and a fifteen pounds unintentional weight-loss, prompting his physician to order a chest $\mathrm{x}$-ray showing extensive bilateral interstitial and airspace infiltrates. A pulmonary function test (PFT) with poor test quality showed FEV1 $11 \%$ predicted and FEV1/FEV 72\% predicted. A positive IgG test for coccidioides prompted a pulmonary coccidioidomycosis diagnosis. Referred to a pulmonologist and a computed tomography (CT) scan showed chronic extensive interstitial, air space, and groundglass densities with granulomatous disease in the mediastinum and hilar regions. A bronchoscopy with a lung biopsy was peformed with negative results. Short after, he was admitted with worsening dyspnea on exertion and had a right thoracoscopy with lung wedge resections. He was diagnosed with disseminated pulmonary coccidioidomycosis and was treated appropriately. Pathology report showed acute on chronic lung injury with fibrosis, microscopic honeycombing and foreign body reaction. During hospital stay he mentioned to his physician that he worked for seventeen years in a golf clubs factory that used beryllium to make lightweight golf clubs, including nine years of working with beryllium. He did not wear a respirator or receive training about beryllium. A revision of the lung pathology histological examination showed interstitial pneumonitis with numerous Schaumann bodies and occasional non-necrotizing granulomas, consistent with berylliosis. A Beryllium Lymphocyte Proliferation Test (BeLPT) showed abnormal lymphocyte transformation to beryllium sulfate, confirming the diagnosis. A follow up PFT showed severe obstructive lung defect. His respiratory symptoms continued to worsen requiring him to use continuous oxygen of 2-4 litres. Two years later he had to get lungs transplant due to pulmonary system failure with good results. After finding out his disease was work-related, his brother-in-law, a 50-year-old male who is symptoms-free and with no significant medical history, and had worked in the same factory in the putter grinding department for six years, reported his occupational history to his personal physician. He had the BeLPT performed with positive result, diagnosis him with BeS. CT scan showed residual of old granulomatous disease with multiple calcified small pulmonary nodules and calcified nodes. The patient is with no symptoms related to BeS.

Discussion In this report, we describe two beryllium golf clubs manufacturing workers who developed BeS and CBD. Beryllium is a metal that is extremely light and strong with high melting point and a high thermal and electrical conductivity making it suitable for a wide range of industries, including defense, aerospace, nuclear, and electronics. Its lightweight makes it fitting for producing lightweight golf clubs. About 62000 workers in the U.S. are exposed to beryllium in their workplaces and the risk of developing $\mathrm{BeS}$ and/or CBD from occupational beryllium exposure has been recognised for a long time. The risk factors include individual genetic susceptibility and the amount of beryllium exposure. The proportion of workers with $\mathrm{BeS}$ that progress to $\mathrm{CBD}$ varies widely, ranging from $9 \%$ to $100 \%$ with CBD developing approximately 10-20 years after first exposure. The importance of protecting workers from beryllium exposure is evident with OSHA's recent lowering of the permissible exposure limit (PEL) for beryllium to $0.2 \mathrm{mcg} / \mathrm{m}^{3}$ air, averaged over 8 hours in hope to save workers from BeS and CBD. With several case reports showing BeS development after a brief or opportunistic exposure to beryllium in administrative staff, it is important to minimise or eliminate beryllium exposure. Workers with Beryllium exposure need ongoing medical screening and surveillance for BeS.

Conclusion These two cases underline the critical need to protect workers from harmful exposure to beryllium, using engineering and work practice controls along with personal protective equipment. For $\mathrm{BeS}$ and $\mathrm{CBD}$, it is recommended to eliminate or minimise further exposure to beryllium. 